\title{
Notizie
}

\section{CIO - Convegno italiano di ornitologia}

Il ventesimo convegno nazionale degli ornitologi italiani si terrà a Napoli, dal 25 al 29 settembre 2019, organizzato dal CISO (Centro Italiano Studi Ornitologici) e da ARDEA (Associazione per la Ricerca, la Divulgazione e l'Educazione Ambientale).

Il programma si articolerà in una serie di sessioni scientifiche, workshop tematici alternati con numerosi momenti sociali - ricreativi. Tutte le informazioni e i moduli per le iscrizioni sono disponibili sul sito WEB del Convegno e annunciati nelle circolari. Il sito web del convegno è in costante aggiornamento ed è opportuno visitarlo spesso: www.xxcio.ardeaonlus.it

Le sessioni scientifiche saranno le seguenti:

- Uccelli e cambiamenti globali: dalle trasformazioni ambientali al cambiamento climatico

- I monitoraggi

- La gestione dell'avifauna

- L'impatto del cambiamento ambientale globale sugli uccelli da preda

- La conservazione degli uccelli

- Ecologia del movimento degli uccelli

- Gli avvoltoi in Italia, status, minacce e conservazione

- La conservazione degli uccelli attraverso i progetti LIFE: successi e criticità di recenti esperienze italiane

- Ornitologia in laboratorio

- Movimenti degli uccelli veleggiatori: relazioni sociali, volo, vento e suolo

- Ecologia e comportamento

Sono anche previste delle sessioni per i poster e delle tavole rotonde.

L'apertura delle registrazioni dei partecipanti avverrà Mercoledì 25 Settembre 2019. Le sessioni scientifiche cominceranno Giovedì 26 Settembre e si concluderanno Sabato 28 Settembre. Il 29 Settembre sarà dedicato alle escursioni. 